\title{
Linking Processing Parameters and Morphological Development in Organic Photovoltaics by Energy-Filtered TEM Imaging of Model Multilayer Structures
}

\author{
A. A. Herzing, H. W. Ro, and D. M. DeLongchamp
}

Material Measurement Laboratory, National Institute of Standards and Technology, 100 Bureau Drive, Gaithersburg, MD 20899.

Organic photovoltaic (OPV) cells offer a low-cost and lightweight alternative to similar solid state devices. In most cases, high efficiency OPV devices are achieved using a bulk-heterojunction (BHJ) architecture consisting of a three-dimensional (3D), interpenetrating network of donor and acceptor phases (typically a conductive polymer and a fullerene derivative, respectively) [1]. High performance devices must contain an active layer thick enough to absorb the incoming photon flux as well as a nanoscale morphology that facilitates separation of the photon-generated excitons and transport of the resulting charge carriers through the film to the relevant electrode [2]. The active layer is typically fabricated via spin casting, and the resulting nanoscale morphology is strongly influenced by processing parameters such as the choice of solvent, the solvent evaporation rate, and the temperature and duration of any thermal treatment. The complex interplay of these phenomena can be difficult to disentangle, especially in the context of a high-efficiency BHJ architecture. Our group has previously reported the suitability of energy-filtered transmission electron microscopy (EF-TEM) for the characterization of the nanoscale morphology in devices based on poly(3-hexylthiophene) (P3HT) and [6,6]-phenyl-C61-butyric acid methyl ester (PCBM), the most widely studied OPV system [3]. Herein, we report the combined use of focused ion-beam (FIB) sample preparation techniques and valence- and core-loss EF-TEM imaging for investigating fundamental aspects of phase evolution in model, multi-layer specimens of P3HT and PCBM.

Multi-layer specimens were fabricated via sequential spin-casting and mechanical lamination of pure layers of PCBM and P3HT onto a silicon substrate as described previously by Ro et al. [4]. Several designs were investigated, including the tri-layer structure depicted in Figure 1. In this case, two groups of specimens were produced, one which consisted of a layer of P3HT sandwiched between two as-cast (i.e. amorphous) layers of PCBM, and another which was identical except that the bottom-most PCBM layer was pre-crystallized by thermal annealing prior to deposition of the top two layers. These tri-layer specimens were then thermally annealed for various times at $140{ }^{\circ} \mathrm{C}$, which is just above $\mathrm{T}_{\mathrm{g}}$ but below $\mathrm{T}_{\mathrm{m}}$ for both materials. Valence-loss EF-TEM imaging was able to clearly reveal the distribution of both phases, while core-loss mapping of the $\mathrm{C}$-K edge was used to quantitatively assess the change in carbon distribution as a function of heat treatment. In both groups of specimens, uptake of PCBM by the middle P3HT layer occurred after even short annealing times. In the case of the tri-layer containing only amorphous PCBM, a steady state conditions was quickly reached where the net flux across the middle P3HT layer remained constant. However, in the pre-crystallized tri-layer, the less mobile crystalline PCBM acted to anchor the amorphous PCBM which migrated into and through the middle P3HT layer; resulting in the formation of a bi-layer after annealing for $60 \mathrm{~s}$.

In addition to this family of specimens, we will also discuss results obtained from other model multilayer structures designed to investigate the effects of metallization and further thermal treatment conditions. These results have important implications for producing true OPV devices with carefully controlled morphology, and we will demonstrate that the study of model structures with careful 
specimen preparation and spectroscopic imaging is a powerful method for developing structureproperty-processing correlations in OPV technology.

\section{References:}

[1] G. Yu et al., Science, 270 (1995) p. 1789.

[2] C. Brabec et al., Organic Photovoltaics: Concepts and Realization, Springer, Berlin, 2003.

[3] A.A. Herzing, L.J. Richter and I.M. Anderson, J. Phys. Chem. C 114 (2010) p. 17501.

[4] H. W. Ro et al., Macromolecules 45 (2012) p. 6587.

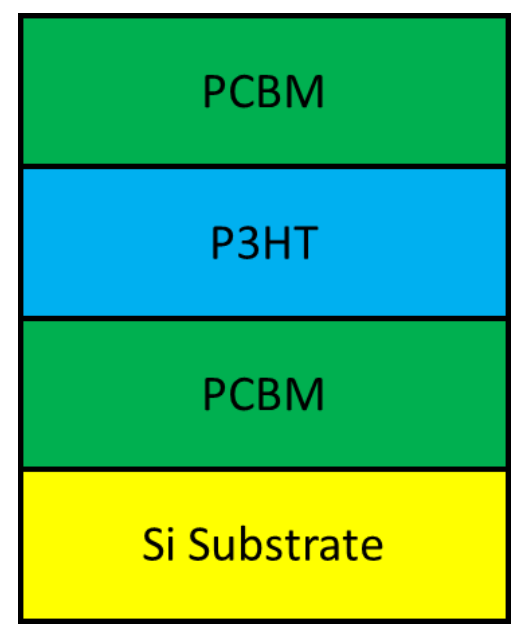

Figure 1 - Schematic showing the design of one of the model tri-layer structures studied in this work. A single layer of P3HT is sandwiched between two PCBM layers.

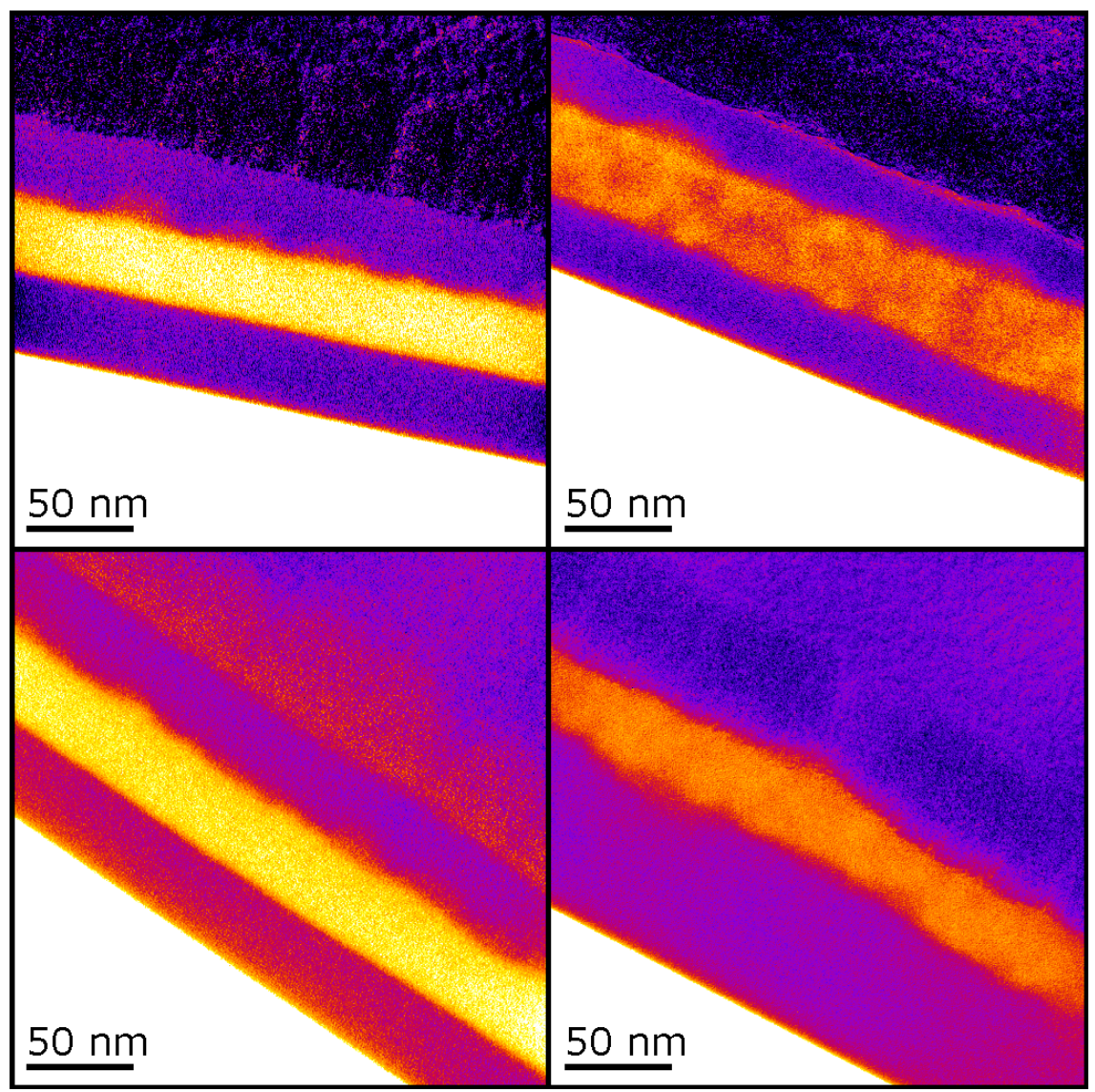

Figure 2 - False-color, low-loss EF-TEM images showing the distribution of P3HT (bright) and PCBM (dark) domains as a function of heat treatment time. 